\title{
Vertex Domination in Fuzzy Graphs
}

\author{
Mohammadesmail Nikfar \\ Independent Scholar \\ nikfarmath1@gmail.com
}

\begin{abstract}
We introduce a new variation on the domination theme which we call vertex domination as reducing waste of time in transportation planning and optimization of transport routes. We determine the vertex domination number $\gamma_{v}$ for several classes of fuzzy graphs. The bounds is obtained for it. In fuzzy graphs, monotone decreasing property and monotone increasing property are introduced. We prove both of the vizing's conjecture and the Grarier-Khelladi's conjecture are monotone decreasing fuzzy graph property for vertex domination. We obtain Nordhaus-Gaddum (NG) type results for these parameters. The relationship between several classes of operations on fuzzy graphs with the vertex domination number of them is studied. Finally, we discuss about vertex dominating set of a fuzzy tree by using the bridges and $\alpha$-strong edges equivalence.
\end{abstract}

Keywords: fuzzy graph, fuzzy bridge, fuzzy tree, $\alpha$-strong arc, vertex domination AMS Subject Classification: 05C72, 05C69, 03E72, 94D05

\section{Introduction}

In 1965, Zadeh published his seminal paper "fuzzy sets" (Ref. [40]) as a way for representing uncertainty. In 1975, fuzzy graphs were introduced by Rosenfeld (Ref. [39]) and Yeh and Bang (Ref. [40]) independently as fuzzy models which can be used in problems handling uncertainty. In 1998, the concept of domination in fuzzy graphs was introduced by A. Somasundaram and S. Somasundaram (Ref. [35]) as the classical problems of covering chess board with minimum number of chess pieces. They defined domination in fuzzy graph by using effective edges (Refs. [35] and [36]). The works on domination in fuzzy graphs were also done such as domination (Refs. [19] and [27]), strong domination (Refs. [18] and [21]), (1,2)-vertex domination (Ref. [34]), 2-domination (Ref. [28]), connected domination (Ref. [20]), total domination (Ref. [22]), Independent domination (Ref. [30]), Complementary nil domination (Ref. [13]), Efficient domination (Ref. [38]), strong (weak) domination (Ref. [29]) and etc.

The rest of this paper is organized as follows. In Section 2, we lay down the preliminary results which recall some basic concepts of fuzzy graph, path, cycle, connectedness, Types of arcs consists of $\alpha$-strong, $\beta$-strong, $\delta$-strong, strong and $M$-strong, complete fuzzy graph, order, size, complement, bipartite fuzzy graph, complete bipartite fuzzy graph, star fuzzy graph, be isolated, some operations on fuzzy graphs consists of cartesian product, join and union and finally we conclude this section with Remark (2.1) and In Section 3, The vertex domination number of a fuzzy graph is defined in a classic way, Definition (3.7), (3.9), (3.10). We determine vertex domination number for several classes of fuzzy graphs consists of complete fuzzy graph, Proposition 
(3.16), empty fuzzy graph, Proposition (3.17), star fuzzy graph, Proposition (3.19), complete bipartite fuzzy graph, Proposition (3.20). We give an upper bound for the vertex domination number of fuzzy graphs, Proposition (3.30). For any fuzzy graph the Nordhaus-Gaddum(NG)'s result holds, Theorem (3.31). Finding domatic partition of size two in fuzzy graph $G$ of order $n \geq 2$ is studied, Theorem (3.34). We improve upper bound for the vertex domination number of fuzzy graphs without isolated nodes, Theorem (3.35). We also improve Nordhaus-Gaddum(NG)'s result for fuzzy graphs without isolated nodes, Corollary (3.36). We give the relationship between $M$-strong arcs and $\alpha$-strong arcs, Corollary (3.38). We give a necessary and sufficient condition for vertex domination which is half of order, In fact fuzzy graphs with vertex domination which is half of order is characterized in the special case, Theorem (3.40). The bridges and $\alpha$-strong edges are equivalence, Corollary (3.23). Some results about fuzzy forest, fuzzy trees and the vertex dominating sets of them is studied, Corollary (3.28), Proposition (3.29).The vertex domination of union of two fuzzy graphs is studied, Proposition (3.41). Also the vertex domination of union of fuzzy graphs family is discussed, Corollary (3.42). The concepts of both monotone increasing fuzzy graph property, Definition (3.43), and monotone decreasing fuzzy graph property, Definition (3.45), are introduced. The result in relation with vizing's conjecture by using $\alpha$-strong arc and monotone decreasing fuzzy graph property is determined, Theorem (3.48). Some results in relation with vizing's conjecture by using $\alpha$-strong arc and spanning fuzzy subgraph is studied, Corollary (3.49). The vertex domination of join of two fuzzy graphs is studied, Proposition (3.50). Also the vertex domination of join of fuzzy graphs family is discussed, Corollary (3.51). The result in relation with Gravier and Khelladi's conjecture by using $\alpha$-strong arc and monotone decreasing fuzzy graph property is determined, Theorem (3.52). We conclude this section with some result in relation with Gravier and Khelladi's conjecture by using $\alpha$-strong arc and spanning fuzzy subgraph, Corollary (3.53). In Section 4, We give one practical application in relation with these concepts.

\section{Preliminary}

We provide some basic background for the paper in this section.

We shall now list below some basic definitions and results of crisp graph, fuzzy subset and fuzzy graph from [4], [31], [26], respectively.

we lay down the preliminary results which recall some basic concepts of fuzzy graph, path, cycle, connectedness, types of arcs consists of $\alpha$-strong, $\beta$-strong, $\delta$-strong, strong and $M$-strong, complete fuzzy graph, order, size, complement, bipartite fuzzy graph, complete bipartite fuzzy graph, star fuzzy graph, be isolated, some operations on fuzzy graphs consists of cartesian product, join and union and finally we conclude this section with Remark (2.1)

We concern with a fuzzy graph which is defined on a crisp graph. So we recall the basic concepts of crisp graph.

A graph (Ref. [4], p. 1) $G$ is a finite nonempty set of objects called vertices (the singular is vertex) together with a (possibly empty) set of unordered pairs of distinct vertices of $G$ called edges. The vertex set of $G$ is denoted by $V(G)$, while the edge set is denoted by $E(G)$.

We recall that a fuzzy subset in Ref. ( [31], Definition 1.2.1, p. 3) of a set $S$ is a function of $S$ into the closed interval [0,1].

we lay down the preliminary results which recall some basic concepts of fuzzy graph from Ref. [26].

A fuzzy graph in Ref. ( [26], Section 2, p. 19) is denoted by $G=(V, \sigma, \mu)$ such that $\mu(\{x, y\}) \leq \sigma(x) \wedge \sigma(y)$ for all $x, y \in V$ where $V$ is a vertex set, $\sigma$ is a fuzzy subset of $V$, 
$\mu$ is a fuzzy relation on $V$ and $\wedge$ denote the minimum. We call $\sigma$ the fuzzy vertex set of $G$ and $\mu$ the fuzzy edge set of $G$, respectively. We consider fuzzy graph $G$ with no loops and assume that $V$ is finite and nonempty, $\mu$ is reflexive (i.e., $\mu(\{x, x\})=\sigma(x)$, for all $x$ ) and symmetric (i.e., $\mu(\{x, y\})=\mu(\{y, x\})$, for all $x, y \in V)$. In all the examples $\sigma$ and $\mu$ is chosen suitably. In any fuzzy graph, the underlying crisp graph is denoted by $G^{*}=(V, E)$ where $V$ and $E$ are domain of $\sigma$ and $\mu$, respectively. The fuzzy graph $H=(\tau, \nu)$ is called a partial fuzzy subgraph of $G=(\sigma, \mu)$ if $\nu \subseteq \mu$ and $\tau \subseteq \sigma$. Similarly, the fuzzy graph $H=(\tau, \nu)$ is called a fuzzy subgraph of $G=(V, \sigma, \mu)$ induced by $P$ in if $P \subseteq V, \tau(x)=\sigma(x)$ for all $x \in P$ and $\nu(\{x, y\})=\mu(\{x, y\})$ for all $x, y \in P$. For the sake of simplicity, we sometimes call $H$ a fuzzy subgraph of $G$. We say that the partial fuzzy subgraph $(\tau, \nu)$ spans the fuzzy graph $(\sigma, \mu)$ if $\sigma=\tau$. In this case, we call $(\tau, \nu)$ a spanning fuzzy subgraph of $(\sigma, \mu)$.

For the sake of simplicity, we sometimes write $x y$ instead of $\{x, y\}$.

A path $P$ of length $n$ in is a sequence of distinct vertices $u_{0}, u_{1}, \cdots, u_{n}$ such that $\mu\left(u_{i-1}, u_{i}\right)>0, i=1,2, \cdots, n$ and the degree of membership of a weakest edge is defined as its strength. If $u_{0}=u_{n}$ and $n \geq 3$ then $P$ is called a cycle and $P$ is called a fuzzy cycle, if it contains more than one weakest edge. The strength of a cycle is the strength of the weakest edge in it. The strength of connectedness between two vertices $x$ and $y$ in is defined as the maximum of the strengths of all paths between $x$ and $y$ and is denoted by $\mu_{G}^{\infty}(x, y)$.

A fuzzy graph $G=(V, \sigma, \mu)$ is connected if for every $x, y$ in $V, \mu_{G}^{\infty}(x, y)>0$.

Note that $\mu_{G^{\prime}}(x, y)$ is the strength of connectedness between $x$ and $y$ in the fuzzy graph obtained from $G$ by deleting the arc $x y$. An arc $x y$ in $G$ is $\alpha$-strong if $\mu(x y)>\mu_{G^{\prime}}(x, y)$. An arc $x y$ in $G$ is $\beta$-strong if $\mu(x y)=\mu_{G^{\prime}}(x, y)$. An arc $x y$ in $G$ is $\delta$-arc if $\mu(x y)<\mu_{G^{\prime}}(x, y)$. An arc $x y$ is a strong arc if it is either $\alpha$-strong or $\beta$-strong. An arc $u v$ of a fuzzy graph is called an $M$-strong arc, In order to avoid confusion with the notion of strong arcs, we shall call strong in the sense of Mordeson as M-strong, if $\mu(u v)=\sigma(u) \wedge \sigma(v)$. If $\mu(u v)>0$, then $u$ and $v$ are called neighbors. The set of all neighbors of $u$ is denoted by $N(u)$. Also $v$ is called $\alpha$-strong neighbor of $u$ if arc $u v$ is $\alpha$-strong. The set of all $\alpha$-strong neighbors of $u$ is denoted by $N_{s}(u)$. The degree of a node $v$ is defined as $d(v)=\Sigma_{u \neq v} \mu(u v)$. The $\alpha$-strong degree of a node $v \in V$ is defined as the sum of membership values of all $\alpha$-strong arcs incident at $v$. It is denoted by $d_{s}(v)$. That is $d_{s}(v)=\Sigma_{u \in N_{s}(v)} \mu(u v) . v$ is called effective neighbor of $u$ if arc $u v$ is $M$-strong. The set of all $M$-strong neighbors of $u$ is denoted by $N_{e}(u)$. The $M$-strong degree of a node $v \in V$ is defined as the sum of membership values of all $M$-strong arcs incident at $v$. It is denoted by $d_{e}(v)$. That is $d_{e}(v)=\Sigma_{u \in N_{e}(v)} \mu(u v)$.

A fuzzy graph $G$ is said complete in Ref. ( [26], Definition 2.3, p. 28) if $\mu(u v)=\sigma(x) \wedge \sigma(y)$. for all $u, v \in V$.

The order $p$ and size $q$ of a fuzzy graph $G=(V, \sigma, \mu)$ are defined $p=\Sigma_{x \in V} \sigma(x)$ and $q=\Sigma_{x, y \in V} \mu(x y)$. The scalar cardinality of $S$ is defined to be $\Sigma_{v \in S} \sigma(v)$ and it is denoted by $|S|_{s}$.

The complement of a fuzzy graph $G$, denoted by $\bar{G}$ is defined to $\bar{G}=(V, \sigma, \bar{\mu})$ where $\bar{\mu}(x y)=\sigma(x) \wedge \sigma(y)-\mu(x y)$ for all $x, y \in V$.

A fuzzy graph $G$ is said bipartite if the vertex set $V$ can be partitioned into two nonempty sets $V_{1}$ and $V_{2}$ such that $\mu\left(v_{1} v_{2}\right)=0$ if $v_{1}, v_{2} \in V_{1}$ or $v_{1}, v_{2} \in V_{2}$. Moreover, if $\mu(u v)=\sigma(u) \wedge \sigma(v)$ for all $u \in V_{1}$ and $v \in V_{2}$ then $G$ is called a complete bipartite fuzzy graph and is denoted by $K_{\sigma_{1}, \sigma_{2}}$, where $\sigma_{1}$ and $\sigma_{2}$ are respectively the restrictions of $\sigma$ to $V_{1}$ and $V_{2}$. In this case, If $\left|V_{1}\right|=1$ or $\left|V_{2}\right|=1$ then the complete bipartite fuzzy graph is said a star fuzzy graph which is denoted by $K_{1, \sigma}$.

A node $u$ is said isolated if $\mu(u v)=0$ for all $v \neq u$.

Now, we will define some special operations on fuzzy graphs. The pages of references will show the proof of validity of them. 
The cartesian product in Ref. ( [25], Proposition 2.1, pp. 160, 161) $G=G_{1} \times G_{2}$ of ${ }_{125}$ two fuzzy graphs $G_{i}=\left(V_{i}, \sigma_{i}, \mu_{i}\right), i=1,2$ is defined as a fuzzy graph $G=\left(V \times V, \sigma_{1} \times \sigma_{2}, \mu_{1} \times \mu_{2}\right)$ where

$E=\left\{\left\{u u_{2}, u v_{2}\right\} \mid u \in V_{1}, u_{2} v_{2} \in E_{2}\right\} \cup\left\{\left\{u_{1} w, v_{1} w\right\} \mid u_{1} v_{1} \in E_{1}, w \in V_{2}\right\}$. Fuzzy sets

$\sigma_{1} \times \sigma_{2}$ and $\mu_{1} \times \mu_{2}$ are defined as $\left(\sigma_{1} \times \sigma_{2}\right)\left(u_{1}, u_{2}\right)=\sigma_{1}\left(u_{1}\right) \wedge \sigma_{2}\left(u_{2}\right)$ and

$\forall u \in V_{1}, \forall u_{2} v_{2} \in E_{2},\left(\mu_{1} \times \mu_{2}\right)\left(\left\{u u_{2}, u v_{2}\right\}\right)=\sigma_{1}(u) \wedge \mu_{2}\left(u_{2} v_{2}\right)$ and

$\forall u_{1} v_{1} \in E_{1}, \forall w \in V_{2},\left(\mu_{1} \times \mu_{2}\right)\left(\left\{u_{1} w, v_{w}\right\}\right)=\mu_{1}\left(u_{1} v_{1}\right) \wedge \sigma_{2}(w)$.

The union $G=G_{1} \cup G_{2}$ in Ref. ( [25], Proposition 3.1, pp. 166, 167) of two fuzzy graphs $G_{i}=\left(V_{i}, \sigma_{i}, \mu_{i}\right), i=1,2$ is defined as a fuzzy graph

$G=\left(V_{1} \cup V_{2}, \sigma_{1} \cup \sigma_{2}, \mu_{1} \cup \mu_{2}\right)$ where $E=E_{1} \cup E_{2}$. Fuzzy sets $\sigma_{1} \cup \sigma_{2}$ and $\mu_{1} \cup \mu_{2}$ are defined as $\left(\sigma_{1} \cup \sigma_{2}\right)(u)=\sigma_{1}(u)$ if $u \in V_{1}-V_{2},\left(\sigma_{1} \cup \sigma_{2}\right)(u)=\sigma_{2}(u)$ if $u \in V_{2}-V_{1}$, and $\left(\sigma_{1} \cup \sigma_{2}\right)(u)=\sigma_{1}(u) \vee \sigma_{2}(u)$ if $u \in V_{1} \cap V_{2}$. Also $\left(\mu_{1} \cup \mu_{2}\right)(u v)=\mu_{1}(u v)$ if

$u v \in E_{1}-E_{2}$ and $\left(\mu_{1} \cup \mu_{2}\right)(u v)=\mu_{2}(u v)$ if $u v \in E_{2}-E_{1}$, and

$\left(\mu_{1} \cup \mu_{2}\right)(u v)=\mu_{1}(u v) \vee \mu_{2}(u v)$ if $u v \in E_{1} \cap E_{2}$.

Let $G=G_{1}+G_{2}$ denote the join in Ref. ( [25], Proposition 3.3, p. 168) of two fuzzy graphs $G_{i}=\left(V_{i}, \sigma_{i}, \mu_{i}\right), i=1,2$ is defined as a fuzzy graph $G=\left(V_{1} \cup V_{2}, \sigma_{1}+\sigma_{2}, \mu_{1}+\mu_{2}\right)$ where $E=E_{1} \cup E_{2} \cup E^{\prime}$ and $E^{\prime}$ is the set of all edges joining vertices of $V_{1}$ with the vertices of $V_{2}$, and we assume that $V_{1} \cap V_{2}=\emptyset$. Fuzzy sets $\sigma_{1}+\sigma_{2}$ and $\mu_{1}+\mu_{2}$ are defined as $\left(\sigma_{1}+\sigma_{2}\right)(u)=\left(\sigma_{1} \cup \sigma_{2}\right)(u)$ and

$\forall u \in V_{1} \cup V_{2} ;\left(\mu_{1}+\mu_{2}\right)(u v)=\left(\mu_{1} \cup \mu_{2}\right)(u v)$ if $u v \in E_{1} \cup E_{2}$ and $\left(\mu_{1}+\mu_{2}\right)(u v)=\sigma_{1}(u) \wedge \sigma_{2}(v)$ if $u v \in E^{\prime}$.

Remark 2.1. For the sake of simplicity, we do sometimes saying $\sigma(x)$ and $\mu(x y)$ with different literature, e.g. value, weight, membership value and etc.

\section{Main Results}

In this section, we provide the main results.

In what follows, the motivation of this research work is presented.

At first, we compare our new definition with previous definitions about domination in fuzzy graphs. We do this comparison on constructing both of "number" and "set" by attention to mathematical concepts and applications. Finally, we give mathematical definitions together some examples which are used them. From the mathematical aspects, being equivalent the $\alpha$-strong arcs with the bridges, Corollary (3.23), cause which we use $\alpha$-strong arcs for constructing a $\alpha$-strong dominating set. The bridges have deeply concepts and various results in fuzzy graph theory due to their definition which show that they are important arcs. These arcs are also related to many important concepts of other fuzzy graphs areas, e.g. fuzzy forest, fuzzy tress, fuzzy cut node, fuzzy cut arcs and etc. Definition of this concept state that those arcs are changing of strength of connectedness which is very important from theoretical and applicational aspects. Because the sensitive roads are effective. These roads will change any decision about transportation in reality. These arcs are definitely interesting in the context of networks, the realization that networks are everywhere is fundamental to our modern lives. It becomes even more important now that algorithms are becoming more and more prevalent in everything too. Speaking of understanding proteins is a an example. Analyzing networks, e.g. molecular networks, food web network, protein interactions network, facebook and other dense networks, for the realization of networks are "everywhere". From social networks such as facebook, the world wide web and the internet to the complex interactions between proteins in the cells of your bodies, we face the challenge of understanding their structure and developments. We are also interesting in the research works in new technologies that can make the future or make the future better as possible. In reality, if we have a set of cities, then those have various roads which have various types of both of qualities and numbers. Quality of 
locations is different. We use "bridges" which are sensitive paths for "constructing the set" of dominating locations and also use "quality of locations" together quality of sensitive paths and all paths for "constructing the number" of domination of locations on other locations. In other words, we construct a new fuzzy graph from previous fuzzy graph model by assigning a new values with respect to summation their initial values with a fraction from values of sensitive roads to values of all roads. We want to decrease the costs. So this number must be the minimum, i.e. we must use the locations and the roads which have the less values as possible for selection of the set of interesting locations. The membership function $\sigma$ on the node set of $G$ can be constructed from the statistical data that represents value of cities with respect to population, locations of stations, facilities of stations, speed of doing works, number of stations, weather and climate, uniques properties, available different roads, number of passengers in different seasons, solving special requirements of passengers, busy time and etc. The membership function $\mu$ on the arc set of $G$ can be constructed from the statistical data with respect to less number of crime, accidents, beauty of the roads, suitable weather, lower raining, lower block of the road, lower road events e.g. falling stones, lower snowing, high numbers of less raining days, lower number of warming days, number of emergency locations in the roads, high security in events, quality of facilities in events, lower number of block of the road due to bad weather. Now the terms "lower, high, less, beauty, busy, quality" are vague in nature. Thus we get a fuzzy graph model. It is interesting to note that a road is of some city to next city and a path contains some roads. Now, we opt some roads which have a highest privilege between other paths. In our terminology, we call these roads by $\alpha$-strong arcs. If these roads deleted, the maximum privilege of all paths decrease between two cities. Thus we pay attentions to these special roads. Every city outside of the set of special cities must be connected to at least one special cities by the special road. For constructing the number of this fuzzy model, we assign to each special cities, a new privilege which is obtained from summation its previous privilege with amount of power of privilege of special roads to others. Finally, we opt the set which summation of privilege of its cities are the minimum. We call it by vertex dominating set. We also get a number which state other presentation of this fuzzy model with respect to privileges of cities, privileges of all roads and privileges of all special roads. This number is called by vertex domination number.

Now, we will bring the old definitions which serves as a foundation of the rest comparison with the newest.

In 1998, the concept of effective domination in fuzzy graphs was introduced by A. Somasundaram and S. Somasundaram (Ref. [35]) as the classical problems of covering chess board with minimum number of chess pieces.

Definition 3.1 (Ref. [35], Definition 2.9, p. 3). Let $G=(\sigma, \mu)$ be a fuzzy graph. Then $D \subseteq V$ is said to be effective dominating set, if for every $v \in V-D$, there exists $u$ in $D$ such that $\mu(u v)=\mu(u) \wedge \mu(v)$. Let $S$ be the set of all effective dominating sets in $G$. The effective domination number of $G$ is defined as $\min _{D \in S}\left(\Sigma_{u \in D} \sigma(u)\right)$ and it is denoted by $\gamma(\mathbf{G})$.

In 2010, the concept of 2-strong(weak) domination in fuzzy graphs was introduced by C. Natarajan and S.K. Ayyaswamy (Ref. [29]) as the extension of strong (weak) domination in crisp graphs.

Definition 3.2 (Ref. [29], p. 1035). Let $G=(\sigma, \mu)$ be a fuzzy graph. Then $D \subseteq V$ is said to be 2-strong(weak) dominating set, if for every $v \in V-D$, there exists $u$ in $D$ such that $\mu(u v)=\mu(u) \wedge \mu(v)$ and $d_{e}(u) \geq d_{e}(v)$. Let $S$ be the set of all 2-strong(weak) dominating sets in $G$. The 2-strong(weak) domination number of $G$ is defined as $\min _{D \in S}\left(\Sigma_{u \in D} \sigma(u)\right)$ and it is denoted by $\gamma_{\mathbf{s f}}(\mathbf{G})\left(\gamma_{\mathbf{w f}}(\mathbf{G})\right)$. 
In 2014, the concept of 1-strong domination in fuzzy graphs was introduced by O.T. Manjusha and M.S. Sunitha (Ref. [19]) as the extension of domination in fuzzy graphs with strong arcs.

Definition 3.3 (Ref. [19], Definition 4.1(c), p. 3208). Let $G=(\sigma, \mu)$ be a fuzzy graph. Then $D \subseteq V$ is said to be 1-strong dominating set, if for every $v \in V-D$, there exists $u$ in $D$ such that $\mu(u v) \geq \mu_{G^{\prime}}^{\infty}(u, v)$. Let $S$ be the set of all 1-strong dominating sets in $G$. The 1-strong domination number of $G$ is defined as $\min _{D \in S}\left(\Sigma_{u \in D} \sigma(u)\right)$ and it is denoted by $\gamma_{\mathbf{S n}}(\mathbf{G})$.

In 2015, the concept of 2-domination in fuzzy graphs was introduced by A. Nagoor Gani and K. Prasanna Devi (Ref. [28]) as the extension of 2-domination in crisp graphs.

Definition 3.4 (Ref. [28], Definition 3.1, p. 120). Let $G=(\sigma, \mu)$ be a fuzzy graph. Then $D \subseteq V$ is said to be 2-dominating set, if for every $v \in V-D$, there exists two nodes like $u$ in $D$ such that $\mu(u v)=\mu_{G^{\prime}}^{\infty}(u, v)$. Let $S$ be the set of all 2-dominating sets in $G$. The 2-domination number of $G$ is defined as $\min _{D \in S}\left(\Sigma_{u \in D} \sigma(u)\right)$ and it is denoted by $\gamma_{\mathbf{2}}(\mathbf{G})$.

In 2015, the concept of strong domination in fuzzy graphs was introduced by O.T. Manjusha and M.S. Sunitha (Ref. [18]) as reduction of the value of old domination number and extraction of classic results.

Definition 3.5 (Ref. [18], Definition 3.1, p. 372). Let $G=(\sigma, \mu)$ be a fuzzy graph. Then $D \subseteq V$ is said to be strong dominating set, if for every $v \in V-D$, there exists $u$ in $D$ such that $\mu(u v) \geq \mu_{G^{\prime}}^{\infty}(u, v)$. Let $S$ be the set of all strong dominating sets in $G$.The strong domination number of $G$ is defined as $\min _{D \in S}\left(\Sigma_{u \in D} t(u, v)\right)$ and it is denoted by $\gamma_{\mathbf{s}}(\mathbf{G})$, where $t(u, v)$ is the minimum of the membership values (weights) of the arc $u v$ such that $\mu(u v) \geq \mu_{G^{\prime}}^{\infty}(u, v)$.

In 2016, the concept of $(1,2)$-domination in fuzzy graphs was introduced by $\mathrm{N}$. Sarala and T. Kavitha (Ref. [34]) as the extension of $(1,2)$-domination in crisp graphs.

Definition 3.6 (Ref. [34], Definition 3.1, p. 16502). Let $G=(\sigma, \mu)$ be a fuzzy graph. Then $D \subseteq V$ is said to be $(1,2)$-dominating set, if for every $v \in V-D$, there exists at least one node in $D$ at distance 1 from $v$ and a second node in $D$ at distance almost 2 from $v$. Let $S$ be the set of all $(1,2)$-dominating sets in $G$. The $(1,2)$-domination number of $G$ is defined as $\min _{D \in S}\left(\Sigma_{u \in D} \sigma(u)\right)$ and it is denoted by $\gamma_{(\mathbf{1 , 2})}(\mathbf{G})$.

a few researchers studied other domination variations which are based on above definitions, e.g. connected domination (Ref. [20]), total domination (Ref. [22]), Independent domination (Ref. [30]), Complementary nil domination (Ref. [13]), Efficient domination (Ref. [38]). So we only compare our new definition with the fundamental dominations which are defined above.

The vertex domination number of a fuzzy graph is defined in a classic way, Definition (3.7), (3.9), (3.10) as reducing waste of time in transportation planning and optimization of transport routes.

Definition 3.7. Let $G=(\sigma, \mu)$ be a fuzzy graph on $V$. Let $x, y \in V$. We say that $x$ dominates $y$ in $G$ as $\alpha$-strong if the arc $\{x, y\}$ is $\alpha$-strong.

Example 3.8. Let $(\sigma, \mu)$ be a fuzzy graph. By attention to it In Figure (3), the arcs $v_{2} v_{5}, v_{2} v_{4}, v_{3} v_{4}$ and $v_{1} v_{3}$ are $\alpha$-strong and the $\operatorname{arcs} v_{1} v_{4}, v_{1} v_{2}$ and $v_{4} v_{5}$ are not $\alpha$-strong.

Definition 3.9. A subset $S$ of $V$ is called a $\alpha$-strong dominating set in $G$ if for every $v \notin S$, there exists $u \in S$ such that $u$ dominates $v$ as $\alpha$-strong. 
Definition 3.10. Let $G=(\sigma, \mu)$ be a fuzzy graph. For every $u \in V$, vertex weight of $u$ is defined as $\sigma(u)+\frac{d_{s}(u)}{d(u)}$ and it is denoted by $\mathbf{w}_{\mathbf{v}}(\mathbf{u})$. Natural extension of this concept to a set, is as follows. We also say $\Sigma_{u \in S}\left(w_{v}(u)\right)$, vertex weight of $S$, for every $S \subseteq V$ and it is denoted by $\mathbf{w}_{\mathbf{v}}(\mathbf{S})$. Now, let $U$ be the set of all $\alpha$-strong dominating sets in $G$. The vertex domination number of $G$ is defined as $\min _{D \in U}\left(w_{v}(D)\right)$ and it is denoted by $\gamma_{\mathbf{v}}(\mathbf{G})$. If $d(u)=0$, for some $u \in V$, then we consider $\frac{d_{s}(u)}{d(u)}$ equal with 0 . The $\alpha$-strong dominating set that is correspond to $\gamma_{v}(G)$ is called by vertex dominating set.

Example 3.11. Let $G=(\sigma, \mu)$ be a fuzzy graph. In Figure (3), the set $\left\{v_{2}, v_{3}\right\}$ is the $\alpha$-strong dominating set. This set is also vertex dominating set in fuzzy graph $G$. Hence $\gamma_{v}(G)=1.75+0.9+0.7=3.35$. So $\gamma_{v}(G)=3.35$.

The comparison between old definitions and our new definition about domination in fuzzy graphs can be discussed by structures of terms "dominating set", and "domination number".

Dominating set. The structure of "dominating set" only depend on the type arc which is used in constructing it. We use the type of arc which is equivalent with bridge. This type of arc in comparison to other type arcs which are used in old definition, is more useful from mathematical and applicational perspective as mentioned in the first of this section. Hence these problems cause motivation for us to changing the type of arc which construct "dominating set".

Domination number. "Domination number" are introduced in old definitions, based on either the values of nodes or the values of arcs, however we defined the domination number by both of value of nodes and value of arcs. In old definitions, either the values of locations or the values of path is considered, however these parameters is simultaneously affected on any decision as mentioned in the first of this section. The many variables can be defined for the junctions in planning transportation e.g. Generation variables: Occupation in the residence area, Population, Residential space, Population density, Number of households, Car ownership rate, Average price of one square meter of land, Students population, Traffic zone space, Number of residential buildings, Distance to entertainment complexes. Attraction variables: Occupation in the working area, Business/ Administrative/Agricultural/Industrial Land Space, Administrative building space, Number of Administrative/Business/Industrial Buildings, Schools' space, Number of Students/Schools/Classes, Number of Universities/Students, Number of Retailers, Number and Capacity of Cinemas/Mosques/ Exhibitions,

Parks/Hospitals. These variables can be positive or negative. Vertex weight of a node in a fuzzy graph can be useful. Another privilege of this definition can be another modeling of the situation. We can assign a new value to every junctions by its vertex weight. Now, we have a new fuzzy graph model. In this model, the roads have no values but value of junctions is more useful in transportation planning. We can pay attentions to the cities which have higher value, for assigning assets, optimization of their routes, or planning of travel and transportation. So this motivated us to improve the definition of "domination number".

Applications. Reducing waste of time in transportation planning and optimization of transport routes are examples of importance of these concepts as mentioned in Section (4). A case study on optimization of transport routes is as follows. A bicyclist may prefer a route where the acceptable parts (for this study, acceptable: the output value is over 0.5 ; above the average; the all parts of route are $\alpha$-strong 
by literatures of this research work.) are higher than on other route-referring to 319 Route 2. Hence this study case illustrates the importance of choice of roads type, 320 $\alpha$-strong arcs, which are introduced as the acceptable parts of route in this case. Another ones, Reducing waste of time in transportation planning by using the concept of vertex domination.

The following is a table consist of a brief fundamental comparison between types of Domination in fuzzy graphs.

\begin{tabular}{|c|c|c|}
\hline Notations & The types of arcs & Number \\
\hline$\gamma(\mathbf{G})$ & $M$-strong & Scalar cardinality \\
\hline$\gamma_{\mathbf{s f}}(\mathbf{G})\left(\gamma_{\mathbf{w f}}(\mathbf{G})\right)$ & $M$-strong and $d_{e}(u) \geq d_{e}(v)$ & Scalar cardinality \\
\hline$\gamma_{\mathbf{S n}}(\mathbf{G})$ & strong & Scalar cardinality \\
\hline$\gamma_{\mathbf{2}}(\mathbf{G})$ & $\beta$-strong & Scalar cardinality \\
\hline$\gamma_{\mathbf{s}}(\mathbf{G})$ & strong & $\min _{D \in S}\left(\Sigma_{u \in D} t(u, v)\right)$ \\
\hline$\gamma_{(\mathbf{1}, \mathbf{2})}(\mathbf{G})$ & Distance & Scalar cardinality \\
\hline$\gamma_{\mathbf{v}}(\mathbf{G})$ & $\alpha$-strong & $\min _{D \in S}\left[\Sigma_{u \in D}\left(\sigma(u)+\frac{d_{s}(u)}{d(u)}\right)\right]$ \\
\hline
\end{tabular}

Two different examples of complete bipartite fuzzy graphs are the following which compare types of Domination in fuzzy graphs.
Figure 1. Comparison of dominations

Figure 2. Comparison of dominations with different values

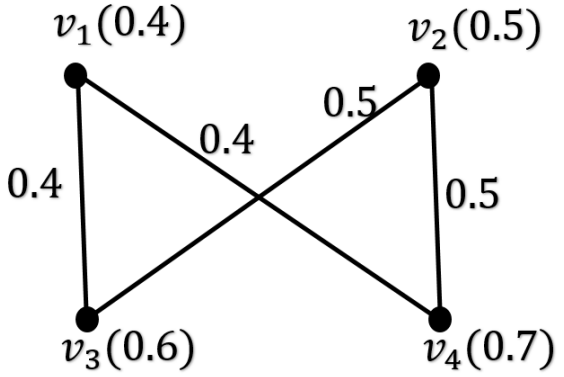

Example 3.12. We use 8

different types of domination on the complete bipartite fuzzy graph $K_{2,2}$ in figure (1). In this fuzzy graph model, we have $\gamma_{s}=0.4, \gamma_{S n}=0.9$, $\gamma_{M}=1, \gamma_{(1,2)}=0.9$

$\gamma_{2}=2.2=p, \gamma_{s f}=1.2$,

$\gamma_{w f}=1, \gamma_{v}=2.2$.

Second example illustrates which our domination number has different value from the others.

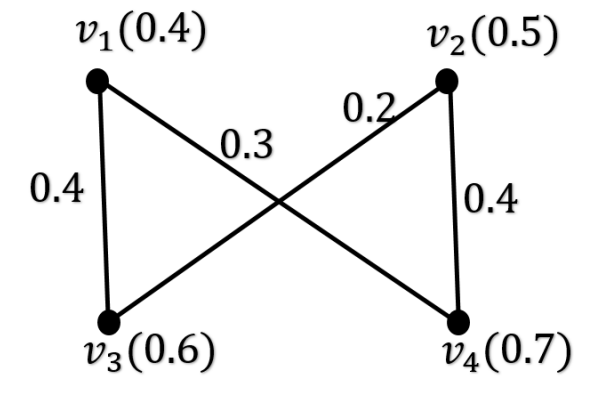

Example 3.13. Again, we use $8 \quad 341$ different types of domination on that different complete bipartite fuzzy grapłas $K_{2,2}$ in figure (2). In this fuzzy model $_{344}$ we have $\gamma_{s}=0.2, \gamma_{S n}=1.6$, $\gamma_{M}=0.9, \gamma_{(1,2)}=0.9$ $\gamma_{2}=2.2=p, \gamma_{s f}=1.1$, $\gamma_{w f}=1.1, \gamma_{v}=2.1$.

It is well known and generally accepted that the problem of determining the domination number of an arbitrary graph is a difficult one. Because of this, researchers have turned their attention to the study of classes of graphs for which the domination problem can be solved in polynomial time.

We determine vertex domination number for several classes of fuzzy graphs consists of complete fuzzy graph, Proposition (3.16), empty fuzzy graph, Proposition (3.17), star fuzzy graph, Proposition (3.19), complete bipartite fuzzy graph, Proposition (3.20).

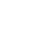


Theorem 3.14. If $G$ is a complete fuzzy graph such that there exists exactly one path with strength of $\mu^{\infty}(u, v)$, then all arcs are $\alpha$-strong.

Proof. Let $G=(\sigma, \mu)$ be a complete fuzzy graph, i.e. $K_{\sigma}$. The strength of path $P$ from $u$ to $v$ is of the form $\sigma(u) \wedge \cdots \wedge \sigma(v) \leq \sigma(u) \wedge \sigma(v)=\mu(u v)$. So $\mu^{\infty}(u, v) \leq \mu(u v)$. $u v$ is a path from $u$ to $v$ such that $\mu(u v)=\sigma(u) \wedge \sigma(v)$. So $\mu^{\infty}(u, v) \geq \mu(u v)$. Hence $\mu^{\infty}(u, v)=\mu(u v)$. Hence $\mu(u v)>\mu^{\prime}(u, v)$. It means the arc $u v$ is $\alpha$-strong. The result follows.

Theorem 3.15. If $G$ is a complete bipartite fuzzy graph such that there exists exactly one path with strength of $\mu^{\infty}(u, v)$, then all arcs are $\alpha$-strong.

Proof. Let $G=(\sigma, \mu)$ be a complete bipartite fuzzy graph, i.e. $K_{\sigma_{1}, \sigma_{2}}$. All arcs of $K_{\sigma_{1}, \sigma_{2}}$ are only from $V_{1}$ to $V_{2}$. Also, this fuzzy graph is complete. Hence the theorem is proved by analogous to proof of Theorem (3.14).

Proposition 3.16 (Complete fuzzy graph). If $G=(V, \sigma, \mu)$ is a complete fuzzy graph, then $\gamma_{v}(G)=\min _{u \in V}(\sigma(u))+1$.

Proof. Since $G$ is a complete fuzzy graph, all arcs are $\alpha$-strong by theorem (3.14) and each node is incident to all other nodes. Hence $D=\{u\}$ is a $\alpha$-strong dominating set and $d_{s}(u)=d(u)$ for each $u \in V$. Hence the result follows.

Proposition 3.17 (Empty fuzzy graph). Let $G=(V, \sigma, \mu)$ be a fuzzy graph. Then $\gamma_{v}(G)=p$, if $G$ be edgeless, i.e $G=\bar{K}_{n}$.

Proof. Since $G$ is edgeless, Hence $V$ is only $\alpha$-strong dominating set in $G$ and none of arcs are $\alpha$-strong. so we have $\gamma_{v}(G)=\min _{D \in S}\left[\Sigma_{u \in D} \sigma(u)\right]=\Sigma_{u \in v} \sigma(u)=p$ by

Definition (3.10). so we can write $\gamma_{v}\left(\bar{K}_{n}\right)=p$ by our notations.

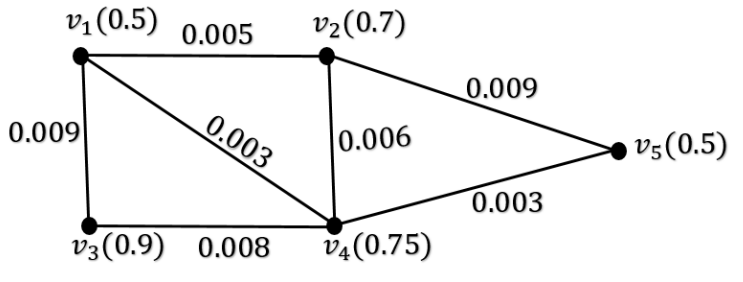

It is interesting to note the converse of Proposition (3.17) that does not hold.

Example 3.18. We show

the converse of Proposition (3.17) does not hold. For this purpose, Let $V=\left\{v_{1}, v_{2}, v_{3}, v_{4}, v_{5}\right\}$. We define $\sigma$ on $V$ by $\sigma: V \rightarrow[0,1]$ such that

Figure 3. Vertex domination

$$
\sigma\left(v_{1}\right)=0.5, \sigma\left(v_{2}\right)=0.7, \sigma\left(v_{3}\right)=0.9, \sigma\left(v_{4}\right)=0.75, \sigma\left(v_{5}\right)=0.5
$$

Now, The function $\mu: V \times V \rightarrow[0,1]$ is defined by

$$
\mu\left(v_{1} v_{2}\right)=0.005, \mu\left(v_{1} v_{4}\right)=0.003, \mu\left(v_{1} v_{3}\right)=0.009, \mu\left(v_{2} v_{4}\right)=0.006, \mu\left(v_{2} v_{5}\right)=0.009 \text {, }
$$

$\mu\left(v_{3} v_{4}\right)=0.008, \mu\left(v_{4} v_{5}\right)=0.003$ such that $\forall u, v \in V, \mu(u, v) \leq \sigma(u) \wedge \sigma(v)$. Finally, Let $V, \sigma$, and $\mu$ be the vertices, value of vertices and value of edges respectively. In other words, By attention to fuzzy graph In Figure (3), the $\operatorname{arcs} v_{2} v_{5}, v_{2} v_{4}, v_{3} v_{4}$ and $v_{1} v_{3}$ are $\alpha$-strong and the $\operatorname{arcs} v_{1} v_{4}, v_{1} v_{2}$ and $v_{4} v_{5}$ are not $\alpha$-strong. So the set $\left\{v_{2}, v_{3}\right\}$ is the $\alpha$-strong dominating set. This set is also vertex dominating set in fuzzy graph $G$. Hence $\gamma_{v}(G)=1.75+0.9+0.7=3.35=\Sigma_{u \in v} \sigma(u)=p$. So $G \neq \bar{K}_{5}$ but $\gamma_{v}(G)=p$.

Proposition 3.19 (Star fuzzy graph). Let $G$ be a star fuzzy graph. Then $G=K_{1, \sigma}$ and $\gamma_{v}\left(K_{1, \sigma}\right)=\sigma(u)+1$ where $u$ is center of $G$. 
Proof. Let $G$ be the star fuzzy graph with $V=\left\{u, v_{1}, v_{2}, \cdots, v_{n}\right\}$ such that $u$ and $v_{i} \quad 389$ are center and leaves of $G$, for $1 \leq i \leq n$ respectively. So $G^{*}=K_{1, n}^{*}$ is underlying crisp graph of $G$. $\{u\}$ is vertex dominating set in $G$ and all arcs are $\alpha$-strong by Theorem (3.15) and due to $G$ is bipartite fuzzy graph. Hence the result follows.

Proposition 3.20 (Complete bipartite fuzzy graph). Let $G$ be the complete bipartite fuzzy graph which is not star fuzzy graph. Then $G=K_{\sigma_{1}, \sigma_{2}}$ and $\gamma_{v}\left(K_{\sigma_{1}, \sigma_{2}}\right)=\min _{u \in V_{1}, v \in V_{2}}(\sigma(u)+\sigma(v))+2$.

Proof. Let $G \neq K_{1, \sigma}$ be bipartite fuzzy graph. Then both of $V_{1}$ and $V_{2}$ include more than one vertex. In $K_{\sigma_{1}, \sigma_{2}}$, all arcs are $\alpha$-strong by Theorem (3.15). Also each node in $V_{1}$ is dominated as $\alpha$-strong with all nodes in $V_{2}$ and conversely. Hence in $K_{\sigma_{1}, \sigma_{2}}$, the $\alpha$-strong dominating sets are $V_{1}$ and $V_{2}$ and any set containing 2 nodes, one in $V_{1}$ and other in $V_{2}$. Hence $\gamma_{v}\left(K_{\sigma_{1}, \sigma_{2}}\right)=\min _{u \in V_{1}, v \in V_{2}}(\sigma(u)+\sigma(v))+2$. So the theorem is proved.

Definition 3.21 (Ref. [26], Section 2.1, p. 21). Let $(\sigma, \mu)$ be a fuzzy graph. Let $x y \in E$. Then $x y$ is called a bridge if $\mu^{\prime}(u, v)<\mu^{\infty}(u, v)$ for some $u, v \in V$, where $\mu^{\prime}(x y)=0$ and $\mu^{\prime}=\mu$ otherwise.

Theorem 3.22 (Ref. [26], Theorem 2.4, pp. 21, 22). Let $(\sigma, \mu)$ be a fuzzy graph. Let $x y \in E$. Let $\mu^{\prime}$ be the fuzzy subset of $E$ such that $\mu^{\prime}(x y)=0$ and $\mu^{\prime}=\mu$ otherwise. Then $(3) \Leftrightarrow(2) \Leftrightarrow(1)$ :

(1) $x y$ is a bridge;

(2) $\mu^{\prime} \infty(x, y)<\mu(x y)$;

(3) $x y$ is not the weakest edge of any cycle.

Corollary 3.23. Let $G=(\sigma, \mu)$ be a fuzzy graph. Let $x y \in E$. xy is a $\alpha$-strong edge if and only if $x y$ is a bridge.

Proof. Obviously, The result is hold by Theorem (3.22).

Proposition 3.24. Let $(\sigma, \mu)$ be a fuzzy graph. If the arc uv is $M$-strong, then $\mu(u v)=\mu^{\infty}(u, v)$.

Proof. Let $G=(\sigma, \mu)$ be a fuzzy graph. The strength of path $P$ from $u$ to $v$ is of the form $\sigma(u) \wedge \cdots \wedge \sigma(v) \leq \sigma(u) \wedge \sigma(v)=\mu(u v)$. So $\mu^{\infty}(u, v) \leq \mu(u v)$. $u v$ is a path from $u$ to $v$ such that $\mu(u v)=\sigma(u) \wedge \sigma(v)$. So $\mu^{\infty}(u, v) \geq \mu(u v)$. Hence $\mu^{\infty}(u, v)=\mu(u v)$. The result follows.

Corollary 3.25. Let $(\sigma, \mu)$ be a fuzzy graph. If the arc uv is $M$-strong such that there exists exactly one path with strength of $\mu^{\infty}(u, v)$. then uv is $\alpha$-strong.

Proof. Let $u v$ be a edge of $(\sigma, \mu)$. So $\mu(u v)=\mu^{\infty}(u, v)$ by Proposition (3.24). Hence $\mu(u v)>\mu^{\prime} \infty(u, v)$. It means the arc $u v$ is $\alpha$-strong.

Definition 3.26 (Ref. [26], Section 2.1, pp. 22, 23). A (crisp) graph that has no cycles is called acyclic or a forest. A connected forest is called a tree. A fuzzy graph is called a forest if the graph consisting of its nonzero edge is a forest and a tree if this graph is also connected. We call the fuzzy graph $G=(\sigma, \mu)$ a fuzzy forest if it has a partial fuzzy spanning subgraph which is a forest, where for all edges $x y$ not in $F[\nu(x y)=0]$, we have $\mu(x y)<\nu^{\infty}(x, y)$. In other words, if $x y$ is in $G$, but not $F$, there is a path in $F$ between $x$ and $y$ whose strength is greater than $\mu(x y)$. It is clear that a forest is a fuzzy forest. If $G$ is connected, then so is $F$ since any arc of a path in $G$ is either in $F$, or can be diverted through $F$. In this case, we call $G$ a fuzzy tree. 
Theorem 3.27 (Ref. [26], Proposition 2.7, p. 24). Let $G=(\sigma, \mu)$ be a fuzzy forest. Then the arcs of $F=(\tau, \nu)$ are just the bridges of $G$.

Corollary 3.28. Let $G=(\sigma, \mu)$ be a fuzzy forest. Then the arcs of $F=(\tau, \nu)$ are just the $\alpha$-strong arcs of $G$.

Proof. Obviously, the results follows by Theorem (3.27) and Corollary (3.23).

Proposition 3.29. Let $T=(\sigma, \mu)$ be a fuzzy tree. Then $D(T)=D(F) \cup D(S)$, where $D(T), D(F)$ and $D(S)$ are vertex dominating sets of $T, F$ and $S$, respectively. $S$ is a set of arcs which has no arcs with connection to $F$.

Proof. By Corollary (3.28), the arcs of $F=(\tau, \nu)$ are just the $\alpha$-strong arcs of $G$. So the result follows by using Definition (3.10).

We give an upper bound for the vertex domination number of fuzzy graphs, Proposition (3.30).

Proposition 3.30. For any fuzzy graph $G=(V, \sigma, \mu)$, We have $\gamma_{v} \leq p$.

Proof. $\gamma_{v}\left(\bar{K}_{n}\right)=p$ by Proposition (3.17). So the result follows.

The classical paper [32] of Nordhaus and Gaddum established the inequalities for the chromatic numbers of a graph $G=(V, E)$ and its complement $\bar{G}$. We are concerned with analogous inequalities involving domination parameters in graphs. We begin with a brief overview of Nordhaus-Gaddum (NG) inequalities for several domination-related parameters. For each generic invariant $\mu$ of a graph $G$, let $\mu=\mu(G)$ and $\bar{\mu}=\mu(\bar{G})$. Inequalities on $\mu+\bar{\mu}$ and $\mu \cdot \bar{\mu}$ exist in the literature for only a few of the many domination-related parameters and most of these results are of the additive form. In 1972 Jaeger and Payan [14] published the first NG results involving domination. Cockayne and Hedetniemi [6] sharpened the upper bound for the sum. Laskar and Peters [17] improved this bound for the case when both $G$ and $\bar{G}$ are connected. A much improved bound was established for the case when neither $G$ nor $\bar{G}$ has isolated nodes by Bollobás and Cockayne [1] and by Joseph and Arumugam [15] independently. For the vertex domination number $\gamma_{v}$ the following theorem gives a Nordhaus-Gaddum type result.

For any fuzzy graph the Nordhaus-Gaddum(NG)'result holds, Theorem (3.31).

Theorem 3.31. For any fuzzy graph $G=(V, \sigma, \mu)$, The Nordhaus-Gaddum result holds. In other words, we have $\gamma_{v}+\overline{\gamma_{v}} \leq 2 p$.

Proof. $G$ is fuzzy graph. So $\bar{G}$ is also fuzzy graph. We implement Theorem (3.30) on $G$ and $\bar{G}$. Then $\gamma_{v} \leq p$ and $\overline{\gamma_{v}} \leq p$. Hence $\gamma_{v}+\overline{\gamma_{v}} \leq 2 p$. So the theorem is proved.

Definition 3.32. A $\alpha$-strong dominating set $D$ is called a minimal $\alpha$-strong dominating set if no proper subset of D is a $\alpha$-strong dominating set.

Theorem 3.33. Let $G$ be a fuzzy graph without isolated nodes. If $D$ is a minimal $\alpha$-strong dominating set then $V-D$ is a $\alpha$-strong dominating set.

Proof. Obvious by attentions to all arcs between two sets which are only $\alpha$-strong.

A domatic partition is a partition of the vertices of a graph into disjoint dominating sets. The maximum number of disjoint dominating sets in a domatic partition of a graph is called its domatic number.

Finding a domatic partition of size 1 is trivial and finding a domatic partition of size 2 (or establishing that none exists) is easy but finding a maximum-size domatic partition (i.e., the domatic number), is computationally hard. Finding domatic partition of size two in fuzzy graph $G$ of order $n \geq 2$ is easy by the following. 
Theorem 3.34. Every connected fuzzy graph $G$ of order $n \geq 2$ has a $\alpha$-strong dominating set $D$ whose complement $V-D$ is also a $\alpha$-strong dominating set.

Proof. Obvious by attentions to $V$ which is $\alpha$-strong dominating set for every connected fuzzy graph and analogous to proof of Theorem (3.33).

We improve upper bound for the vertex domination number of fuzzy graphs without isolated nodes, Theorem (3.35).

Theorem 3.35. For any fuzzy graph $G=(V, \sigma, \mu)$ without isolated nodes, We have $\gamma_{v} \leq \frac{p}{2}$.

Proof. Let $D$ be a minimal dominating set of $G$. Then by Theorem (3.34), V-D is a $\alpha$-strong dominating set of $G$. Then $\gamma_{v}(G) \leq w_{v}(D)$ and $\gamma_{v}(G) \leq w_{v}(V-D)$.

Therefore $2 \gamma_{v}(G) \leq w_{v}(D)+w_{v}(V-D) \leq p$ which implies $\gamma_{v} \leq \frac{p}{2}$. Hence the proof is completed.

We also improve Nordhaus-Gaddum(NG)'s result for fuzzy graphs without isolated nodes, Corollary (3.36).

Corollary 3.36. Let $G$ be a fuzzy graph such that both $G$ and $\bar{G}$ have no isolated nodes. Then $\gamma_{v}+\overline{\gamma_{v}} \leq p$, where $\overline{\gamma_{v}}$ is the vertex domination number of $\bar{G}$. Moreover, equality holds if and only if $\gamma_{v}=\overline{\gamma_{v}}=\frac{p}{2}$.

Proof. By the Implement of Theorem (3.35) on $G$ and $\bar{G}$, we have $\gamma_{v}(G)=\gamma_{v} \leq \frac{p}{2}$, and $\gamma_{v}(\bar{G})=\overline{\gamma_{v}}(G)=\overline{\gamma_{v}} \leq \frac{p}{2}$. So $\gamma_{v}+\overline{\gamma_{v}} \leq \frac{p}{2}+\frac{p}{2}=p$. Hence $\gamma_{v}+\overline{\gamma_{v}} \leq p$.

Suppose $\gamma_{v}=\bar{\gamma}_{v}=\frac{p}{2}$, then obviously $\gamma_{v}+\bar{\gamma}_{v}=p$. Conversely, suppose $\gamma_{v}+\overline{\gamma_{v}} \leq p$. Then we have $\gamma_{v} \leq \frac{p}{2}$ and $\overline{\gamma_{v}} \leq \frac{p}{2}$. If either $\gamma_{v}<\frac{p}{2}$ or $\overline{\gamma_{v}}<\frac{p}{2}$, then $\gamma_{v}+\overline{\gamma_{v}}<p$, which is a contradiction. Hence the only possibility case is $\gamma_{v}=\bar{\gamma}_{v}=\frac{p}{2}$.

Proposition 3.37. Let $G=(V, \sigma, \mu)$ be a fuzzy graph. If all arcs have equal value, the $G$ has no $\alpha$-strong edge.

Proof. Obviously the result is hold by using Definition of $\alpha$-strong arc.

We give the relationship between $M$-strong arcs and $\alpha$-strong arcs, Corollary (3.38).

Corollary 3.38. Let $G=(V, \sigma, \mu)$ be a fuzzy graph. If all arcs are $M$-strong, the $G$ has no $\alpha$-strong edge.

Proof. Obviously the result is hold by using Proposition (3.37).

The following example illustrates this concept.

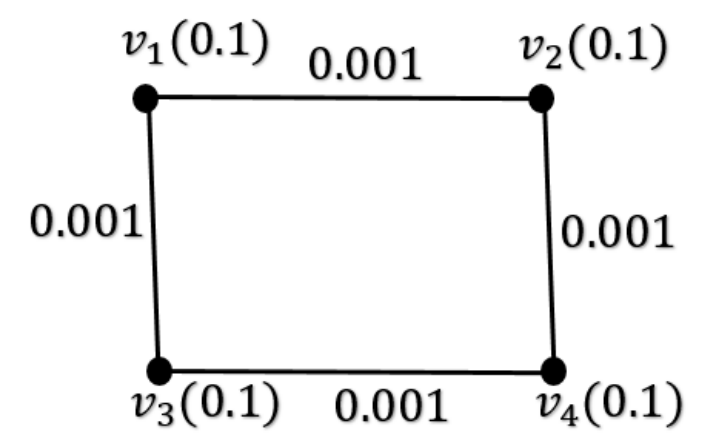

Example 3.39. In Figure

(4), all arcs have the same value but there is no $\alpha$-strong arcs in this fuzzy graph. Obviously this result is hold by using Definition (3.9).

We give a necessary and sufficient condition for vertex domination which is half of order, In fact fuzzy graphs with vertex domination which is half of order is characterized in the special case, Theorem (3.40).
Figure 4. Identical arcs and $\alpha$-strong arcs 
Theorem 3.40. In any fuzzy graph $G=(V, \sigma, \mu)$ such that values of nodes are equal and all arcs have same value, i.e. for $\forall u_{i}, u_{j} \in V$ and $\forall e_{i}, e_{j} \in E$, we have $\sigma\left(u_{i}\right)=\sigma\left(u_{j}\right)$ and $\mu\left(e_{i}\right)=\mu\left(e_{j}\right) . \gamma_{v}=\frac{p}{2}$ if and only if For any vertex dominating set $D$ in $G$, we have $|D|=\frac{n}{2}$.

Proof. Suppose $D$ has the conditions. $d_{s}(D)=0$ by Proposition (3.37). So $\gamma_{v}(G)=\Sigma_{u \in D} \sigma(u)$ by using Definition (3.10). Since values of nodes are equal and $|D|=\frac{n}{2}$, we have $\gamma_{v}(G)=\Sigma_{u \in D} \sigma(u)=\frac{n}{2} \sigma(u)=\frac{1}{2}(n \sigma(u))=\frac{1}{2}\left(\Sigma_{u \in V} \sigma(u)\right)=\frac{1}{2}(p)=\frac{p}{2}$. Hence the result is hold in this case.

Conversely, Suppose $\gamma_{v}=\frac{p}{2}$. Let $D=\left\{u_{1}, u_{2}, \cdots, u_{n}\right\}$ be a vertex dominating set. $d_{s}(D)=0$ by Proposition (3.37). So $\gamma_{v}(G)=\Sigma_{u \in D} \sigma(u)$ by using Definition (3.10). Since $\gamma_{v}(G)=W_{v}(D)$, we have $\gamma_{v}=\frac{p}{2}=\frac{1}{2}\left(\Sigma_{u \in V} \sigma(u)\right)=\Sigma_{u \in D} \sigma(u)$. Suppose $n^{\prime} \neq \frac{n}{2}$. so $\left.\Sigma_{i=1}^{n^{\prime \prime}} \sigma\left(v_{i}\right)\right)=0$ which is a contradiction with $\forall u_{i} \in V, \sigma\left(u_{i}\right)>0$. Hence $n^{\prime}=\frac{n}{2}$, i.e. $|D|=n^{\prime}=\frac{n}{2}$. So the result is hold in this case.

The vertex domination of union of two fuzzy graphs is studied, Proposition (3.41).

Proposition 3.41. Let $G_{1}$ and $G_{2}$ be fuzzy graphs. The vertex dominating set of $G_{1} \cup G_{2}$ is $D=D_{1} \cup D_{2}$ such that $D_{1}$ and $D_{2}$ are the vertex dominating set of $G_{1}$ and $G_{2}$ respectively. Moreover, $\gamma_{v}\left(G_{1} \cup G_{2}\right)=\gamma_{v}\left(G_{1}\right)+\gamma_{v}\left(G_{2}\right)$.

Proof. Obviously the result is hold by using Definition of union of two fuzzy graphs. $(3.42)$.

Also the vertex domination of union of fuzzy graphs Family is discussed, Corollary

Corollary 3.42. Let $G_{1}, G_{2}, \cdots, G_{n}$ be fuzzy graphs. The vertex dominating set of $\cup_{i=1}^{n} G_{i}$ is $D=\cup_{i=1}^{n} D_{i}$ such that $D_{i}$ is the vertex dominating set of $G_{i}$. Moreover, $\gamma_{v}\left(\cup_{i=1}^{n} G_{i}\right)=\sum_{i=1}^{n} \gamma_{v}\left(G_{i}\right)$.

Proof. Obviously the result is hold by using proposition (3.41).

The concepts of both monotone increasing fuzzy graph property, Definition (3.43), and monotone decreasing fuzzy graph property, Definition (3.45), are introduced.

Definition 3.43. We call a fuzzy graph property $P$ monotone increasing if $G \in P$ implies $G+e \in P$, i.e., adding an edge e to a fuzzy graph $G$ does not destroy the property.

Example 3.44. Connectivity and Hamiltonicity are monotone increasing properties. A monotone increasing property is nontrivial if the empty fuzzy graph $\bar{K}_{\sigma} \notin P$ and the complete fuzzy graph $K_{\sigma} \in P$.

Definition 3.45. A fuzzy graph property is monotone decreasing if $G \in P$ implies $G-e \in P$, i.e., removing an edge from a graph does not destroy the property.

Example 3.46. Properties of a fuzzy graph not being connected or being planar are examples of monotone decreasing fuzzy graph properties.

Remark 3.47. Obviously, a fuzzy graph property $\mathrm{P}$ is monotone increasing if and only if its complement is monotone decreasing. Clearly not all fuzzy graph properties are monotone. For example having at least half of the vertices having a given fixed degree $d$ is not monotone. 
In graph theory, Vizing's conjecture [5] concerns a relation between the domination number and the cartesian product of graphs. This conjecture was first stated by Vadim G. Vizing (1968), and states that, if $\gamma(G)$ denotes the minimum number of vertices in a dominating set for $\mathrm{G}$, then

$$
\gamma(G \times H) \geq \gamma(G) \gamma(H)
$$

Vizing's conjecture from 1968 asserts that the domination number of the Cartesian product of two graphs is at least as large as the product of their domination numbers.

Let $\gamma(G)$ denote the domination number of a simple graph $G$. Then Vizing (1963) [5] conjectured that $\gamma(G) \gamma(H) \leq \gamma(G \times H)$, where $G \times H$ is the graph product. While the full conjecture remains open, Clark and Suen (2000) [33] have proved the looser result $\gamma(G) \gamma(H) \leq 2 \gamma(G \times H)$.

Vizing stated the still open conjecture:

Conjecture (Vizing [5]). For all graphs $G$ and $H, \gamma(G) \gamma(H) \leq \gamma(G \times H)$.

The result in relation with vizing's conjecture by using $\alpha$-strong arc and monotone decreasing fuzzy graph property is determined, Theorem (3.48).

Theorem 3.48. The vizing's conjecture is monotone decreasing property in fuzzy graph $G$, if the edge $e$ be $\alpha$-strong and $\gamma_{v}(G-e)=\gamma_{v}(G)$.

Proof. The fuzzy graph $(G-e) \times H$ is the spanning fuzzy subgraph of $G \times H$, for all fuzzy graph $H$. So $\gamma_{v}((G-e) \times H) \geq \gamma_{v}(G \times H) \geq \gamma_{v}(G) \gamma_{v}(H)=\gamma_{v}(G-e) \gamma_{v}(H)$. Hence vizing's conjecture is also hold for $G-e$. Then the result follows.

Some results in relation with vizing's conjecture by using $\alpha$-strong arc and spanning fuzzy subgraph is studied, Corollary (3.49).

Corollary 3.49. Suppose the vizing's conjecture is hold for $G$. Let $K$ be the spanning fuzzy subgraph of $G$ such that $\gamma_{v}(K)=\gamma_{v}(G)$. Then the vizing's conjecture is hold for K.

Proof. The fuzzy graph $K \times H$ is the spanning fuzzy subgraph of $G \times H$, for all fuzzy graph $H$. So $\gamma_{v}(K \times H) \geq \gamma_{v}(G \times H) \geq \gamma_{v}(G) \gamma_{v}(H)=\gamma_{v}(K) \gamma_{v}(H)$. Hence the vizing's conjecture is also hold for $K$. So the result follows.

The vertex domination of join of two fuzzy graphs is studied, Proposition (3.50).

Proposition 3.50. Let $G_{1}$ and $G_{2}$ be fuzzy graphs. The vertex dominating set of $G_{1} \otimes G_{2}$ is $D=D_{1} \cup D_{2}$ such that $D_{1}$ and $D_{2}$ are the vertex dominating set of $G_{1}$ and $G_{2}$ respectively. Moreover, $\gamma_{v}\left(G_{1} \otimes G_{2}\right)=\gamma_{v}\left(G_{1}\right)+\gamma_{v}\left(G_{2}\right)$.

Proof. Obviously the result is hold by using Definition of join of two fuzzy graphs which state in this case, $M$-strong arcs between two fuzzy graphs is not $\alpha$-strong which is weak arc changing strength of connectedness of $G$.

Also the vertex domination of join of fuzzy graphs Family is discussed, Corollary $(3.51)$.

Corollary 3.51. Let $G_{1}, G_{2}, \cdots, G_{n}$ be fuzzy graphs. The vertex dominating set of $\otimes_{i=1}^{n} G_{i}$ is $D=\otimes_{i=1}^{n} D_{i}$ such that $D_{i}$ is the vertex dominating set of $G_{i}$. Moreover, $\gamma_{v}\left(\otimes_{i=1}^{n} G_{i}\right)=\Sigma_{i=1}^{n} \gamma_{v}\left(G_{i}\right)$.

Proof. Obviously the result is hold by using proposition (3.50). 
Gravier and Khelladi (1995) conjectured a similar bound for the domination number of the tensor product of graphs; however, a counterexample was found by Klav ̌ar Zmazek (1996) [16]. Since Vizing proposed his conjecture, many mathematicians have worked on it, with partial results described below. For a more detailed overview of these results, see Brešar et al. (2012) [2]

Gravier and Khelladi [40] conjecture a Vizing-like inequality for the domination number of the cross product of graphs.

Gravier and Khelladi stated the still open conjecture:

Conjecture (Gravier and Khelladi [40]). For all graphs $G$ and $H$,

$$
\gamma(G) \gamma(H) \leq 2 \gamma(G \otimes H) .
$$

The result in relation with Gravier and Khelladi's conjecture by using $\alpha$-strong arc and monotone decreasing fuzzy graph property is determined, Theorem (3.52).

Theorem 3.52. The Gravier and Khelladi's conjecture is monotone decreasing property in fuzzy graph $G$, if the edge e be $\alpha$-strong and $\gamma_{v}(G-e)=\gamma_{v}(G)$.

Proof. The fuzzy graph $(G-e) \times H$ is the spanning fuzzy subgraph of $G \times H$, for all fuzzy graph $H$. So $\gamma_{v}((G-e) \times H) \geq \gamma_{v}(G \times H) \geq \gamma_{v}(G) \gamma_{v}(H)=\gamma_{v}(G-e) \gamma_{v}(H)$. Hence Gravier and Khelladi's conjecture is also hold for $G-e$. Then the result follows.

We conclude this section with some result in relation with Gravier and Khelladi's conjecture by using $\alpha$-strong arc and spanning fuzzy subgraph is studied, Corollary $(3.53)$.

Corollary 3.53. Suppose the Gravier and Khelladi's conjecture is hold for $G$. Let $K$ be the spanning fuzzy subgraph of $G$ such that $\gamma_{v}(K)=\gamma_{v}(G)$. Then the Gravier and Khelladi's conjecture is hold for $K$.

Proof. The fuzzy graph $K \times H$ is the spanning fuzzy subgraph of $G \times H$, for all fuzzy graph $H$. So $\gamma_{v}(K \otimes H) \geq \gamma_{v}(G \otimes H) \geq \gamma_{v}(G) \gamma_{v}(H)=\gamma_{v}(K) \gamma_{v}(H)$. Hence the Gravier and Khelladi's conjecture is also hold for $K$. So the result follows.

\section{Practical Application}

In this section, we introduce three applications are related with transportation planning.

Nowadays, researchers have two main problems. On the one hand people are dependent on critical systems such as transportation, electricity, water supply, sewage, ICT. According to the official definition, the critical infrastructure is a term used to describe assets that are essential for the functioning of a society and economy. The following facilities are related to this subject [7]:

- electricity and heating generation, transmission and distribution;

- gas and oil production, transport and distribution;

- telecommunication;

- water supply;

- agriculture, food production and distribution;

- public health (hospitals, ambulances); 
- transportation systems (fuel supply, railway network, airports, harbours, inland shipping);

- financial services (banking);

- security services (police, military).

There are several regional critical-infrastructure protection programmes, which main aims are:

- to indentify important assets,

- to analyze a risk based on major threat scenarios and the vulnerability of each asset,

- to indentify, select and make prioritisation of counter-measures and procedures.

These goals are common for all facilities presented above. It is very important to keep these systems in good conditions [11,12]. Thus, the risk and reliability analyses is needed to understand the impact of threats and hazards [8,9,11]. Unfortunately, these problems are more and more complex, because of existing strong interdependencies both within and between infrastructure systems. The second problem is finding optimal solutions. It is met in many areas of modern science, technology and economics. For example, the navigator's main aim is optimizing the route of the ship due to safety, time of passage, fuel and costs [24,37].

Transportation planning is a wide human-oriented field with diverse and challenging problems waiting to be solved. Characteristics and performances of transport systems-services, costs, infrastructures, vehicles and control systems are usually defined on the basis of quantitative evaluation of their main effects. Most of the transport decisions take place under imprecision, uncertainty and partial truth. Some objectives and constraints are often difficult to be measured by crisp values. Traditional analytical techniques were found to be non-effective when dealing with problems in which the dependencies between variables are too complex or ill-defined. Moreover, hard computing models can not deal effectively with the transport decision-makers' ambiguities and uncertainties. Trip generation defines that how many people want to go out of their homes and what their purposes are. One of the most important problems in the field of transportation planning is the problem of Origin-destination estimation from link counts. In order to decrease the cost of passenger surveys, traffic count are undertaken on certain links of the transportation network.Trip distribution models are used to determine the number of trips between pairs of zones when the number of trips generated attracted by particular zones is known. Thus, the prediction of trip distribution involves the prediction of flows in a network regardless of a possible transportation mode or travel route. Traffic flows and trip distribution resulted from human choices that are affected by social and individual variables of the commuters. Due to this fact that human decision making are more consistent with fuzzy logic in comparison with crisp mathematics, it seems that fuzzy logic could be a logical tool to map such areas.

-Case study: Reducing waste of time in transportation planning and optimization of transport routes.

At First, we discuss about the various types of parameters which can be a fuzzification of the roads. In other words, We study a fuzzy model of paths between two junctions without considering values of junctions for optimization of transport routes. Finally, We study decreasing waste of time in planning transportation as the main case by the concept of vertex domination.

-Optimization of transport routes as the importance of choice of roads type ( $\alpha$-strong arcs). 
Remark 4.1. For the sake of simplicity, we use the notations "MFs" and "mf" for membership functions and membership function, respectively.

The proposed model is applied to three routes in San Francisco between Golden Gate Bridge and Golden Gate Park. All three routes start from the junction of the Masonic Ave and the McAllister St, stretching to the north end of the Market St. The route is fancied as a bicycling route between the main campus of San Francisco University and the ferry terminal. San Francisco is chosen as the study area, which is the seventh on the list of bicycle commuting rates of 64 U.S. Cities from the Census 2000 Supplemental Survey (C2SS), with 1.80 percent (Dill and Carr, 2003). The city's grid street plan (even though it ignores its irregularly shaped coastline and topography) embodies a rational conception of urban space for bicyclists. San Francisco's mild winters and moderate summers make bicycling easier and less stressful (Pucher, 1999). On the other hand, the hilly terrain of the city, also heavy traffic and lack of bicycle lanes represent unfavorable conditions in the case study. According to the 2008 reports of SFMTA (The San Francisco Municipal Transportation Agency, the majority of the bicycle trips in the city are for utilitarian purposes, to work, school and shopping. Respondents cite the greatest barriers to bicycling as; "not enough bike lanes," "not comfortable biking with cars" and "too difficult to cross major streets". Hills are also mentioned as: "don't want to get sweaty before work". The study by Hood et al. (2011) on route choice in San Francisco indicates that bicyclists prefer bike lanes to other types of bicycle facilities, and dislike climbing hills and turnings. Also bicyclists are not always concerned with taking the shortest routes.

When we set the direction for that origin and destination, Google Maps generates three possible route alternatives as seen in Fig. (5) Route 1 via McAllister St is: $5.5 \mathrm{~km}$, $21 \mathrm{~min}, 13 \mathrm{~m}$ up and $88 \mathrm{~m}$ down in total; Route 2 via Post St is: $5.8 \mathrm{~km}, 23 \mathrm{~min}, 46 \mathrm{~m}$ up and $121 \mathrm{~m}$ down in total; and Route 3 via Pacific Ave is: $7.1 \mathrm{~km}, 30 \mathrm{~min}, 63 \mathrm{~m}$ up and $138 \mathrm{~m}$ down in total. The routes are represented as lines in a continuous blue color on the city map, without expressing the changing conditions of bicycling along the route. Although bicyclists get an idea about approximate travel time and the elevation profile of the complete route, there is no visualization of the path showing the combination of fragmentarily changing data such as the type of bicycle facility and vehicle density on motorways.

For the operational step of the model, the routes are divided into virtual segments by points at every $60-70 \mathrm{~m}$. 85 points for Route $1 ; 87$ points for Route 2 ; and 101 points for Route 3 are pinned on the map. The input values are searched for each point on the line. The inputs: bicycle facility type and vehicle flow (typical traffic, Monday 9 AM) are available in the existing street data in Google Maps. The height (or altitude) is obtained from an elevation finder by giving geographical coordinates of the points. The input: slope is then measured relative to the next point's height. The positive numbers (descending meter) refer to downhill whereas the negative numbers (ascending meter) refer to uphill, and " 0 " means flat surfaces. The variable data of three inputs is listed for all of the points and proceeded as description from Ref. [3]. the results from

Ref. [3] is used for the model with 3 inputs, $332 \mathrm{MFs}, 1$ output and 18 rules with 'and' operator. Inputs are 1. Elevation with MFs: up, straight, down; 2. Road Type with MFs: not defined, bicycle friendly, dedicated lane; 3. Traffic Flow with MFs: slow, fast.

The output quality value in Ref. [3] has six constant membership functions: mf1: 1 (the highest), mf2: 0.8, mf3: 0.6, mf4: 0.4, mf5: 0.2 and mf6: 0 (the lowest). 18 Fuzzy rules are created between inputs and output, as follows: 'If the road is a dedicated lane, elevation is downgrade and traffic is fast, then the bicycle ride quality is mf: 1'. This is the highest result when all the parameters are fully satisfied, and the opposite is 'If the road is unknown, elevation is upgrade and traffic is slow, then the bicycle ride quality is mf: 0 '. The riding quality is evaluated according to all of the three parameters; so while 
two parameters are fully satisfied and one is not satisfied at all, the overall result of that point will decrease.
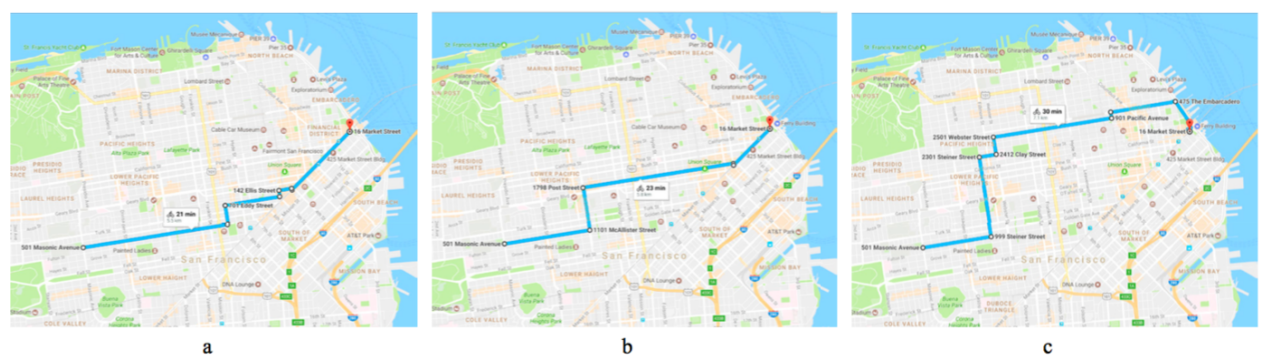

Figure 5. (a) Route 1, (b) Route 2 and (c) Route 3

Each point is calculated in fuzzy operator, and the resulting list of outputs are visualized by coloring the points in accordance with their numeric values. The red part of the scale demonstrates that the bicycle ride quality is very low when bike facility, slope and traffic are in consideration. This also represents the most disadvantageous parts of the route. On the contrary, at the blue part of the color scale, riding quality is high; so the suitability and acceptability of the road is very positive. 1d plots are conveyed on Google Earth, mirroring the points onto their original longitude and latitude, seen in Fig. (6). This map is expected to help bicyclists to see at which parts of the route riding will be more comfortable, safe and easy. And which streets they need to be aware of for a low riding quality; then to pay more attention to the traffic or to be ready for extra physical effort. Additional information of the routes analyses is given in Table (1). The minimum and maximum output values change for three routes. The percentages related to the lines can be useful in bicycling route choose. For instance, Route 1 and Route 2, although they have similar attributes in terms of distance and travel time on Google Maps, show different characteristics. A bicyclist may prefer a route where the acceptable parts (for this study, acceptable: the output value is over 0.5; above the average; the all parts of route are $\alpha$-strong by literatures of this research work.) are higher than on other route-referring to Route 2. Hence this study case illustrates the importance of choice of roads type, $\alpha$-strong arcs, which are introduced as the acceptable parts of route in this case.

\begin{tabular}{lccc}
\hline & Route 1 & Route 2 & Route 3 \\
\hline min. output value & 0.173 & 0.201 & 0.206 \\
max. output value & 0.737 & 0.789 & 0.811 \\
The most advantageous parts; blue (\%) & 24,7 & 10.3 & 16.8 \\
The least advantageous parts; red (\%) & 9.4 & 4.5 & 12.8 \\
The acceptable parts; over 0.5 (\%) & 37.6 & 45.9 & 60.3 \\
\hline
\end{tabular}

Table 1. Results. 

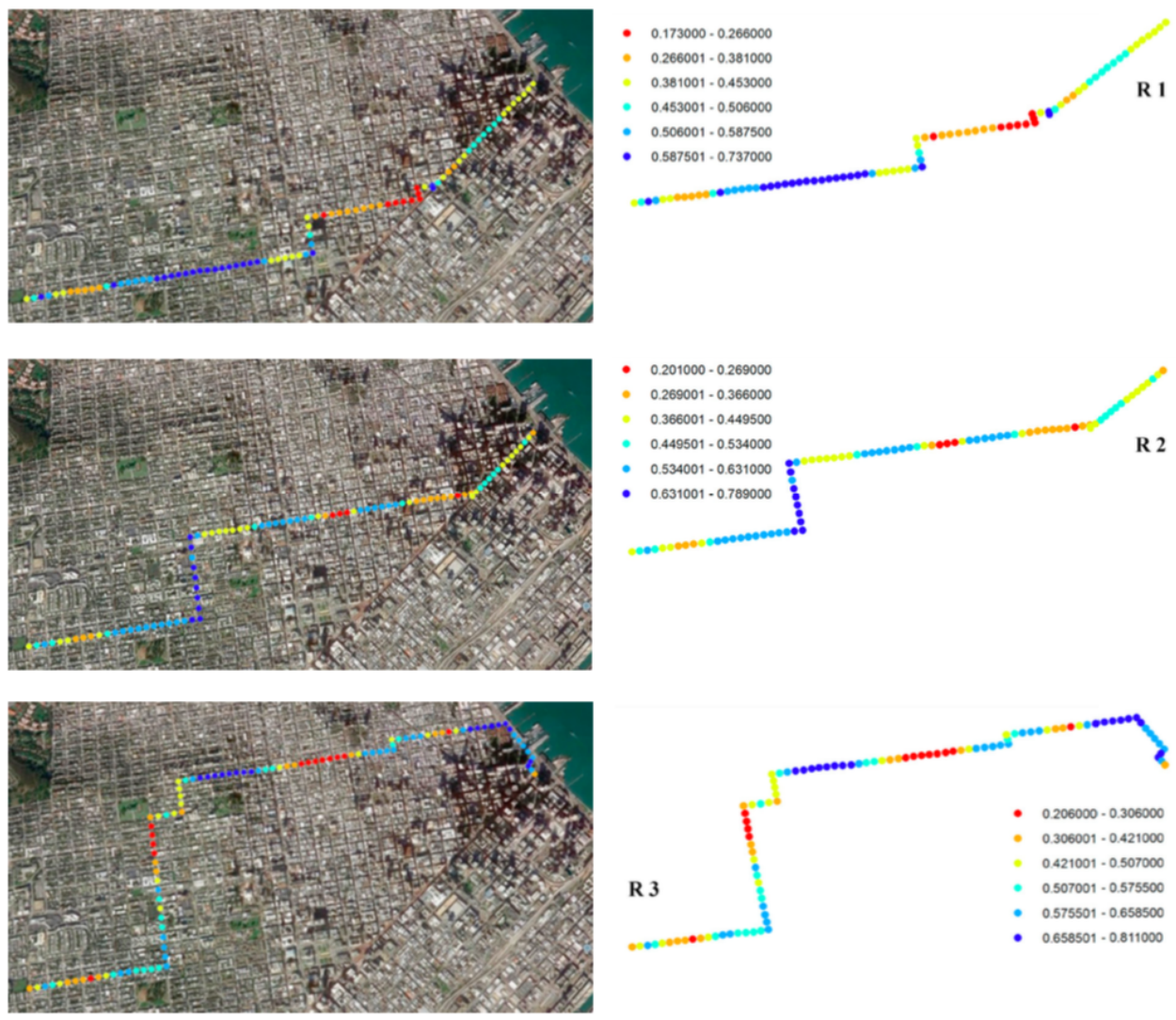

Figure 6. Routes 1,2 and 3 on Google Earth as the results of the model

-Main case study: Reducing waste of time in transportation planning by using the concept of vertex domination

The many variables can be defined for the junctions in planning transportation e.g. Generation variables: Occupation in the residence area, Population, Residential space, Population density, Number of households, Car ownership rate, Average price of one square meter of land, Students population, Traffic zone space, Number of residential buildings, Distance to entertainment complexes. Attraction variables: Occupation in the working area, Business/ Administrative/Agricultural/Industrial Land Space,

Administrative building space, Number of Administrative/Business/Industrial Buildings, Schools' space, Number of Students/Schools/Classes, Number of Universities/Students, Number of Retailers, Number and Capacity of Cinemas/Mosques/ Exhibitions, Parks/Hospitals. These variables can be positive or negative. Let us consider the hypothetic map of road connections, what is given by schema presented in Figure (7). We choose eleven nodes and describe them the consecutive number and the time of red light (in seconds) divided by the maximum of them. The edges between the nodes are described by the number of kilometers divided by the maximum of them. For the sake of simplicity both of calculation and illustration of practical application, we don't affect these divisions on Figure (7). Now, we characterize the $\alpha$-strong arcs which are fundamental for constructing $\alpha$-strong dominating set, in Figure (8). We presented $\alpha$-strong dominating sets in Figures (9) and (10), by using Figure (8). In figure (9), $w_{v}\left(D_{1}\right)=2.52$. In figure (10), $w_{v}\left(D_{2}\right)=5.47$. Finally, the values of nodes, $\alpha$-strong arcs and arcs will present the vertex dominating set and vertex domination number. In this case of study, the vertex dominating set is presented by (11). the vertex domination 
number is $\gamma_{v}(G)=3.17$. The figure (11) is showed that some cities are dominated others as $\alpha$-strong.

Vertex weight of a node in a fuzzy graph can be useful. We can assign a new value to every junctions by its vertex weight. Now, we have a new fuzzy graph model. In this model, the roads have no values but value of junctions is more useful in transportation planning. We can pay attentions to the cities which have higher value, for assigning assets, optimization of their routes, or planning of travel and transportation.

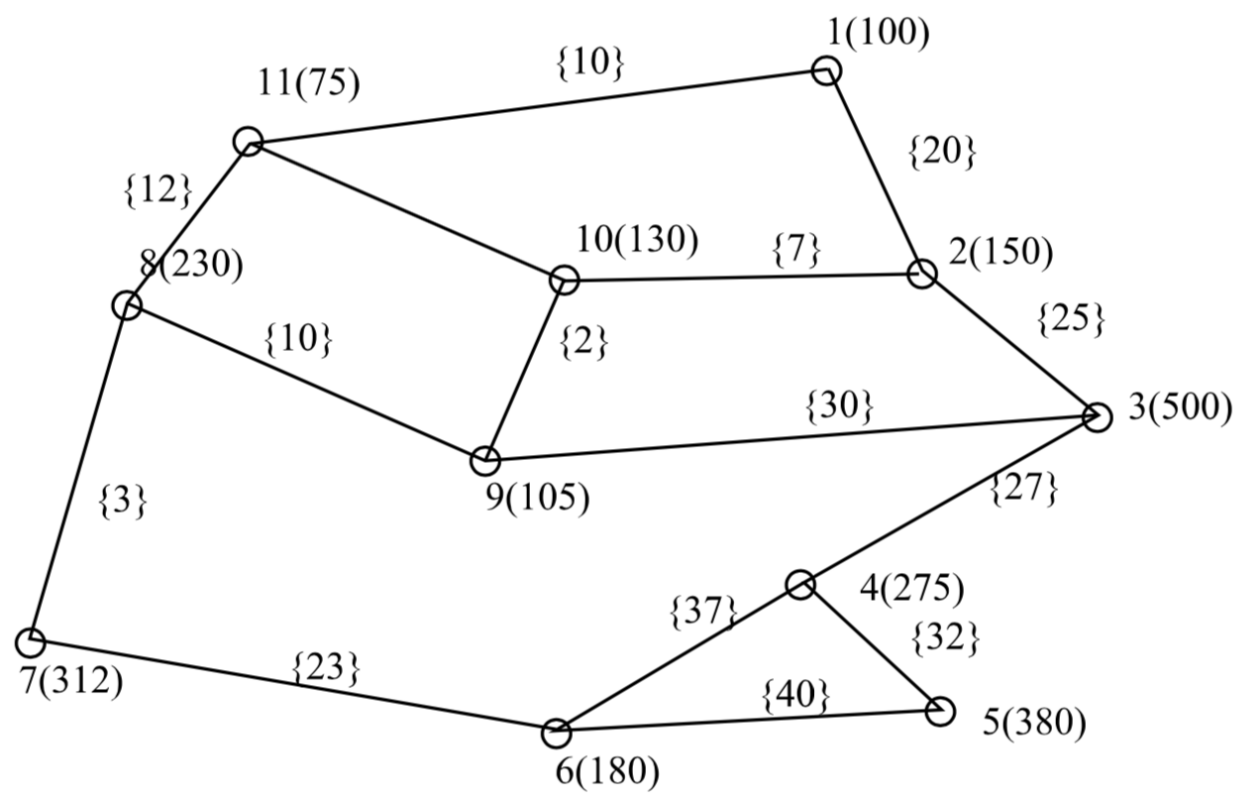

Figure 7. The exemplary scheme of road infrastructure.

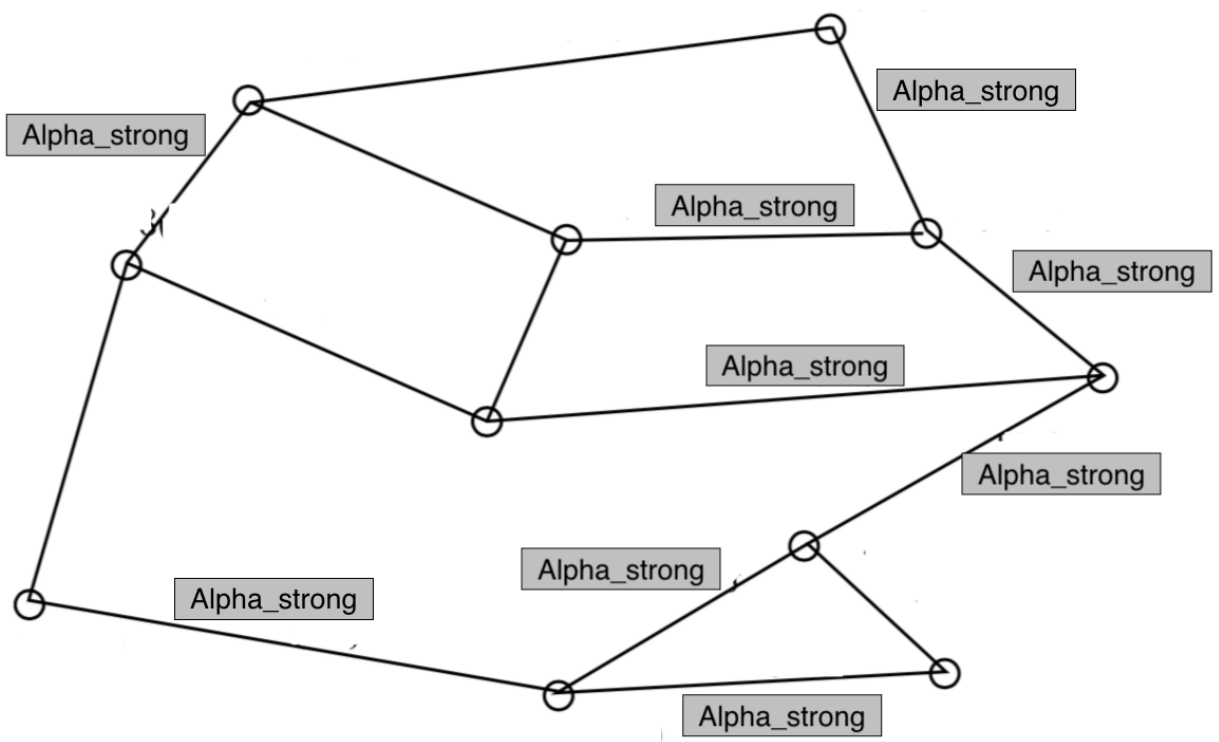

Figure 8. $\alpha$-strong arcs 


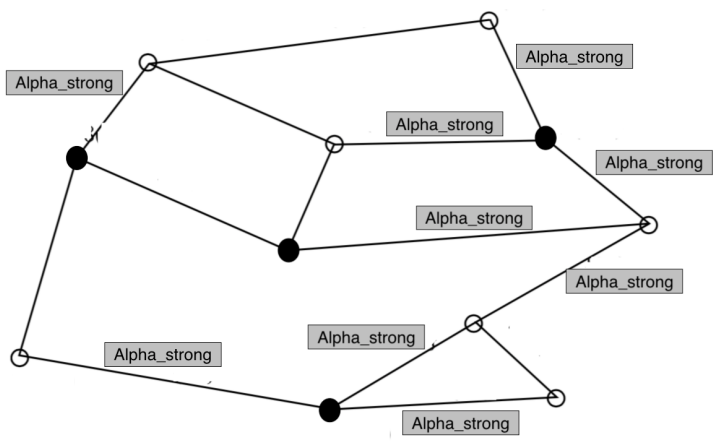

Figure 9. The set of black nodes are $\alpha$-strong dominating set which is called $D_{1}$.

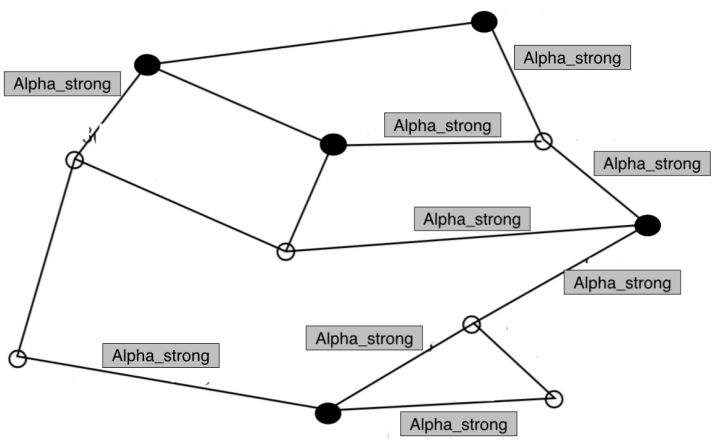

Figure 10. The set of black nodes are $\alpha$-strong dominating set which is called $D_{2}$.

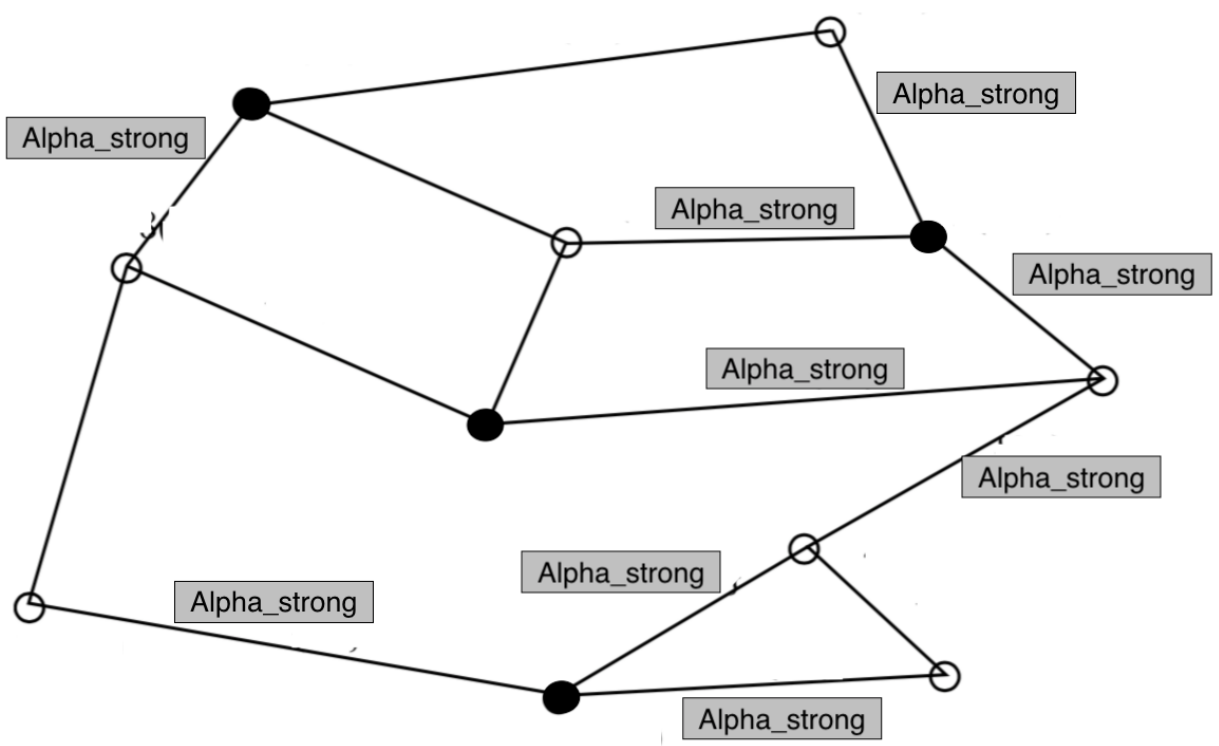

Figure 11. The set of black nodes are vertex dominating set which is called $D$. 


\section{Conclusion}

We introduce a new variation on the domination theme. These concepts are definitely interesting in the context of networks, as mentioned, the realization that networks are "everywhere", is fundamental to our modern lives. It becomes even more important now that algorithms are becoming more and more "prevalent" in everything, too. The mathematical background of this domination are related to other theoretical concepts of fuzzy graphs, more than old definitions. Some applications, from the real-world problems, are better modeled with this definition other than old ones. In one applications, optimization of transport routes occurs such that the acceptable parts are higher than on others. In the other application, reducing waste of time in transportation planning is caused by analyzing data of its fuzzy graph model. From the transport properties, comparison of cities can be better modeled. So we can assign assets usefully or change the infrastructures of transport for reducing waste of time. We hope these concepts are useful for studying problems of mathematics and real-world which make the future better as possible.

\section{Acknowledgments}

We thank just about everybody. The author is highly thankful to the Editor-in-Chief and the referees for their valuable comments and suggestions for improving the paper.

\section{References}

1. B. Bollobás and E.J. Cockayne, unpublished.

2. Brešar, Bos̆tjan; Dorbec, Paul; Goddard, Wayne; Hartnell, Bert L.; Henning, Michael A.; Klavžar, Sandi; Rall, Douglas F. (2012), Vizing's conjecture: a survey and recent results, Journal of Graph Theory 69 (1) 46-76.

3. A. Cekmis, Fuzzy modeling for route characteristic, procedia computer science 120 (2017) 112-119.

4. G. Chartrand and L. Lesniak, Graphs and Digraphs, Third edition, CRC Press, 1996.

5. W.E. Clark and S. Suen, An Inequality Related to Vizing's Conjecture, Electronic J. Combinatorics 71 (2000) 1-3, 2000.

6. E.J. Cockayne and S.T. Hedetniemi, Toward a theory of domination in graphs, Networks 7 (1977) 247-261.

7. COMMISSION OF THE EUROPEAN COMMUNITIES (2006), Communication from the Commission on a European Programme for Critical Infrastructure Protection, Brussels.

8. S. Guze, The graph theory approach to analyze critical infrastructures of transportation systems, Journal of Polish Safety and Reliability Association, Summer Safety and Reliability Seminars, 5 (2) (2014) 57-62.

9. S. Guze and L. Smolarek, (2011). Methods for risk minimizing in the process of decision-making under uncertainty, Journal of Polish Safety and Reliability Association-JPSRA, 2 (1) (2011) 123-128. 
10. S. Guze, Graph Theory Approach to Transportation Systems Design and Optimization, the International Journal on Marine Navigation and Safety of Sea, Transportation 8 (4) (2014) 571-578.

11. K. Kolowrocki, Safety of critical infrastructures. Journal of Polish Safety and Reliability Association, Summer Safety and Reliability Seminars, 4 (1) (2013) 51-74.

12. K. Kolowrocki, Reliability of Large Systems, Elsevier, Amsterdam - Boston Heidelberg - London - New York - Oxford - Paris - San Diego - San Francisco Singapore - Sydney - Tokyo. (2004).

13. M. Ismayil and I. Mohideen, Complementary nil domination in fuzzy graphs, Annals of Fuzzy Mathematics and Informatics (2014) 1-8.

14. F. Jaeger and C. Payan, Relations du type Nordhaus-Gaddum pour le nombre d'absorption d'un graphe simple, C.R. Acad. Sci. Paris A 274 (1972) 728-730.

15. J.P. Joseph and S. Arumugam, A note on domination in graphs, lnternet. J. Management Systems, to appear.

16. S. Klavžar, B. Zmazek, On a Vizing-like conjecture for direct product graphs, Discrete Mathematics, 156 (1996) 243-246.

17. R. Laskar and K. Peters, Vertex and edge domination parameters in graphs, Congr. Numer. 48 (1985) 291-305.

18. O.T. Manjusha and M.S. Sunitha, Strong Domination in Fuzzy Graphs, Fuzzy Inf. Eng. 7 (2015) 369-377.

19. O.T. Manjusha and M.S. Sunitha, Notes on domination in fuzzy graphs, Journal of Intelligent and Fuzzy systems 27 (2014) 3205-3212.

20. O.T. Manjusha and M.S. Sunitha, Connected domination in fuzzy graphs using strong arcs, Annals of Fuzzy Mathematics and Informatics 10 (6) (2015) 979-994.

21. O.T. Manjusha and M.S. Sunitha, The Strong Domination Alteration Sets in Fuzzy Graphs, International Journal of Mathematics and its Applications 4 (2-D) (2016) 109-123.

22. O.T. Manjusha and M.S. Sunitha, Total Domination in Fuzzy Graphs Using Strong Arcs, Annals of Pure and Applied Mathematics 9 (1) (2015) 23-33.

23. S. Mathew and M.S. Sunitha, Types of arcs in a fuzzy graph, Information Sciences 179 (2009) 1760-1768.

24. L. Ming-Hua, T. Jung-Fa, and Y. Chian-Son, A Review of Deterministic Optimization Methods in Engineering and Management, Mathematical Problems in Engineering, (2012).

25. J.N. Mordeson and C.S. Peng, Operations on fuzzy graphs, Information Sciences 79 (1994) 159-170.

26. J.N. Mordeson and P.S. Nair, Fuzzy Graphs and Fuzzy Hypergraphs, Physica-Verlag, 2000.

27. A. Nagoorgani and V.T. Chandrasekaran, Domination in fuzzy graph, Adv. in fuzzy sets and systems 1 (1) (2006) 17-26. 
28. A. Nagoor Gani and K. Prasanna Devi, 2-Domination in Fuzzy Graphs, International Journal of Fuzzy Mathematical Archive 9 (1) (2015) 119-124.

29. C. Natarajan and S.K. Ayyaswamy, on Strong (weak) domination in Fuzzy Graphs, International Journal of Mathematical, Computational, Physical, Electrical and Computer Engineering 4 (7) (2010) 1035-1037.

30. A. Nagoorgani and P.Vadivel, Relations between the parameters of Independent Domination and Irredundance in Fuzzy Graphs, International Journal of Algorithms, Computing and Mathmatics 2 (1) (2009) 15-19.

31. H.T. Nguyen and E.A. Walker, A First course in fuzzy logic, CRC Press, 2006.

32. E.A. Nordhaus and J.W. Gaddum, On complementary graphs, Amer. Math. Monthly 63 (1956) 175-177.

33. A. Rosenfeld, Fuzzy Graphs, In Fuzzy Sets and their Applications to Cognitive and Decision Processes, eds. L.A. Zadeh, K.S. Fu, K. Tanaka and M. Shimura, Academic Press, New York (1975) 77-95.

34. N. Sarala and T. Kavitha, (1,2)-Vertex Domination in Fuzzy Graphs, International Journal of Innovative Research in Science, Engineering and Technology 5 (7) (2016) 16501-16505.

35. A. Somasundaram and S. Somasundaram, Domination in fuzzy graphs-I, Pattern Recognition Letters 19 (1998) 787-791.

36. A. Somasundaram, Domination in fuzzy graphs-II, J. Fuzzy Math. 13 (2) (2005) 281-288.

37. J. Szlapczynska, Multicriteria Evolutionary Weather Routing Algorithm in Practice TransNav, the International Journal on Marine Navigation and Safety of Sea Transportation, 7 (1) (2013) 61-65 Gdynia.

38. Dr.S. Vimala and J.S. Sathya, Efficient Domination number and Chromatic number of a Fuzzy Graph, International Journal of Innovative Research in Science, Engineering and Technology 3 (3) (2014)9965-9970.

39. R.T. Yeh, S.Y. Bang, Fuzzy relations, Fuzzy relations, fuzzy graphs and their applications to clustering analysis, in: Fuzzy Sets and Their Applications to Cognitive and Decision Processes, eds. L.A. Zadeh, K.S. Fu, K. Tanaka and M. Shimura, Academic Press, (1975) 125-149.

40. L.A. Zadeh, Fuzzy sets, Information and Control 8 (1965) 338-353. 